\title{
A COMPREHENSIVE SURVEY OF LOCAL ANAESTHESIA STORAGE AND SAFETY, AND REGIONAL ANAESTHESIA EQUIPMENT IN A MULTIFACETED TERTIARY CENTRE
}

Chaudhary, Atif ${ }^{1}$, Patel, Hinal² ${ }^{2}$ Crowley, Mark ${ }^{3}$; Nuffield Department of Anaesthesia, John Radcliffe, Oxford University Hospital

\section{Background and Aims}

The Royal College of Anaesthetists has recommended guidelines for the safe storage of drugs, and provision of regional anaesthesia, in its Anaesthesia Clinical Services Accreditation framework. Each domain represents gold standard care mapped to the Care Quality Commission's (CQC) 'Key Lines of Enquiry', Health Inspectorate Wales and Health Improvement Scotland inspection domains. These domains are designed to achieve a 'Safe, Effective, Caring, Responsive and Well-Led' Service. We aimed to compare our tertiary referral centre against a target of $100 \%$ compliance as defined by the accreditation scheme.

\section{Methods}

A snapshot survey was performed across 3 hospitals and 44 theatres in our large NHS trust. Each surveyor attended an assigned theatre to individually assess clinical targets against gold-standard care as defined by the Royal College of Anaesthetists. The clinical workload across the hospital sites included Tertiary Orthopaedic, Obstetric, Transplant, Neurosurgical, Cardiothoracic, Paediatric and Bariatric specialties amongst others.

Domains examined included storage of local anaesthesia, availability of equipment (including documentation checklists, ultrasound provision, local anaesthetics, echogenic needles) and knowledge of intralipid, emergency drugs and resuscitation pathways.
Results from a standardized checklist were collated and analysed between sites.

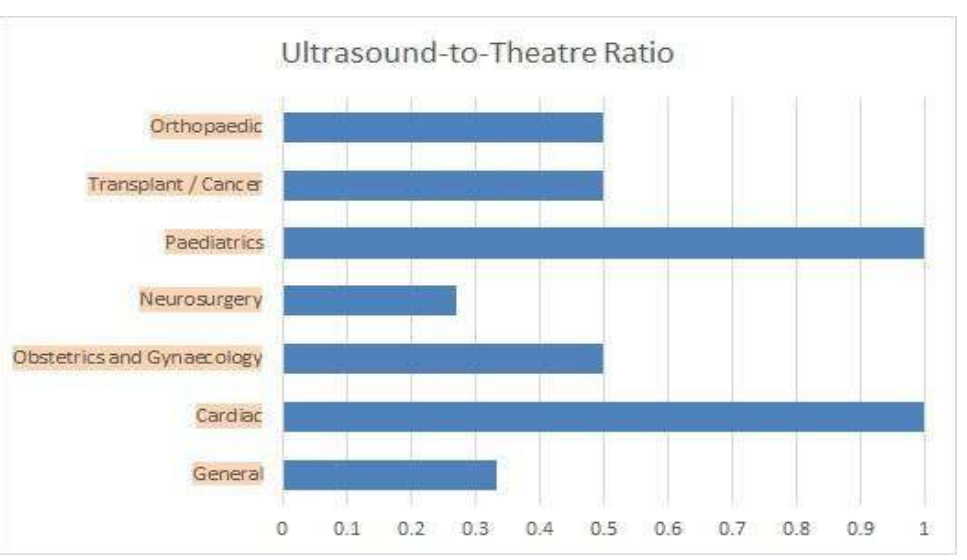

Figure 1: Ultrasound

Results

Our survey demonstrated standardised safety protocols across the trust, with some deficient areas highlighted. Ultrasound machines were available to every site with the lowest ratio of USto-theatre being 1:3.75 (Figure 1)

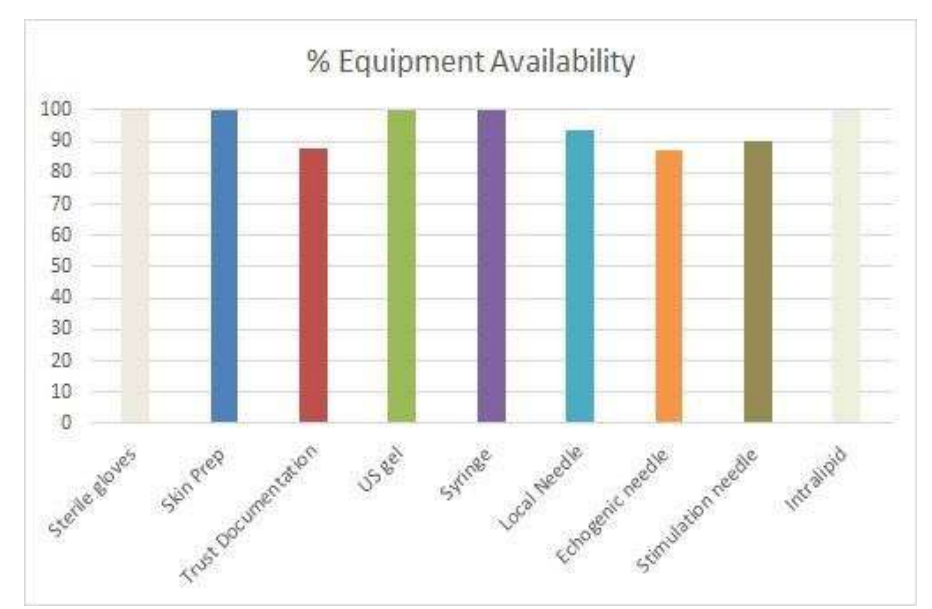
Figure 2: Equipment
Basic and emergency equipment was readily available (Figure 2), with echogenic and nerve stimulator needles available to most but not all - clinical areas. Importantly, the survey highlighted deficiency in local anaesthetic storage with 16/44 theatres storing agents along potentially intravenous administered drugs. The majority of these instances referred to $1 \%$ or $2 \%$ lignocaine in the primary drug cabinet survey have been addressed with education and reorganisation.
Conclusions

Equipment, storage protocols, and practice of regional blocks will naturally vary within a large organisation. Whilst some variation is encouraged (use of different needles, techniques for block), it is important to maintain strict standards of patient safety.

Areas of deficiency highlighted within our 